\title{
Transmisión entre mercados bursátiles y crisis financiera: El caso de España
}

\author{
PAZ Rico BELDA \\ Universidad de Valencia, Facultad CC.EE., Avda. de los naranjos, s/n, 46022 Valencia, España. E- \\ mail: paz.rico@uv.es
}

\begin{abstract}
RESUMEN
El trabajo analiza si la interrelación entre el mercado bursátil español y las bolsas de Estados Unidos, Reino Unido, Alemania y Francia se ha visto afectada y cómo por la reciente crisis financiera. Para ello, se estima un modelo VARGARCH bivariante, durante el período enero de 2000 a junio de 2012. Del modelo estimado se obtiene una medida del grado de integración de los mercados, el coeficiente de correlación condicional, y de éste se concluye que la crisis subprime produjo un efecto contagio entre los mercados bursátiles. Asimismo, la evidencia empírica permite concluir que en el período poscrisis ha aumentado la interrelación de la bolsa española con la francesa, reduciéndose la interdependencia con la británica. Por su parte, la interrelación del mercado español con los mercados alemán y norteamericano ha vuelto a los niveles anteriores a la crisis subprime.
\end{abstract}

Palabras clave: Mercado de valores, interrelación, rendimientos, volatilidad, crisis subprime.

\section{Transmission between Stock Markets and Financial Crisis: The Case of Spain}

\begin{abstract}
The paper analyses if the interrelation between Spanish stock market and stock markets of USA, UK, German and France has been affected, and how, by subprime crisis. For that, a bivariate VAR-GARCH model has been estimated, over the period January 2000 to June 2012. A measure of integration degree, as the conditional correlation coefficient, is obtained from the estimated model. Analysing this measure leads to conclude that subprime crisis had a contagion effect. Moreover, the empirical evidence allows concluding that in poscrisis period the interrelation between Spanish market and French market has increased, while the interrelation with British market has reduced. On the other hand, the interrelation of Spanish stock market with German and USA stock markets have gone back to the level before subprime crisis.
\end{abstract}

Keywords: Stock Markets, Interrelation, Returns, Volatility, Subprime Crisis.

Clasificación JEL: G15, C32

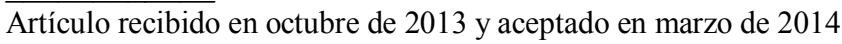

Artículo disponible en versión electrónica en la página www.revista-eea.net, ref. ə-32206 


\section{INTRODUCCIÓN}

La interrelación entre los mercados financieros internacionales resulta de sumo interés, tanto para inversores, académicos y autoridades. Varias son las razones de este interés. En primer lugar, la posibilidad de diversificar el riesgo depende del grado de interrelación entre los mercados ${ }^{1}$. En segundo lugar, si existe una relación de causalidad entre los rendimientos de los mercados financieros, los inversores pueden explotar estrategias de inversión para obtener beneficios en períodos de elevada incertidumbre. En tercer lugar, conocer la interrelación entre los mercados resulta útil en la optimización de carteras y en la fijación del precio de activos. Asimismo, el conocimiento de la interrelación entre los mercados financieros ayuda a las autoridades a controlar los contagios entre mercados, fijando regulaciones efectivas para estabilizar el sistema financiero y los flujos de capital.

Para algunos autores, la integración de los mercados financieros resulta fundamental en el crecimiento económico, al contribuir a la mejora de la eficiencia en la distribución de los recursos y a la reducción de la volatilidad macroeconómica ${ }^{2}$. No obstante, para otros autores, la integración hace que los mercados sean más vulnerables a crisis financieras que ocurren en otras áreas del mundo ${ }^{3}$, y reduce los beneficios de la diversificación internacional ${ }^{4}$.

La interdependencia ${ }^{5}$ entre los mercados bursátiles ha sido objeto de una amplia investigación empírica, desde que Grubel (1968) señaló las ventajas de la diversificación internacional. Los primeros trabajos empíricos se centraban en analizar las relaciones en media entre diferentes mercados ${ }^{6}$, pero a partir de los noventa, se empezó a modelizar la interrelación en segundos momentos. Por otro lado, las metodologías estadísticas y econométricas utilizadas han sido muy variadas ${ }^{7}$. Así, para examinar los co-movimientos entre distintos mercados bursátiles se han empleado desde correlaciones cruzadas ${ }^{8}$, regresiones y contrastes de causalidad $^{9}$, vectores autorregresivos ${ }^{10}$, análisis de

\footnotetext{
${ }^{1}$ Según el CAPM (Capital asset pricing model) el riesgo de mercado nunca se elimina, de tal forma que, para diversificar el riesgo, los inversores deberán invertir en más de un mercado y poner atención en la relación entre los mercados internacionales.

${ }^{2}$ Pagano (1993), Prasad et al. (2003) y Baele et al. (2004).

${ }^{3}$ Büttner y Hayo (2011).

${ }^{4}$ Karim et al. (2011).

${ }^{5}$ Soriano (2004) define interdependiencia como un concepto amplio que recoge todo tipo de relaciones, tanto en media como en varianza, que puede existir entre dos mercados o activos.

${ }^{6}$ Grubel y Fadner (1971)), Agmon (1972) y Lessard (1973).

${ }^{7}$ Para una revisión exhaustiva de la literatura empírica veáse Soriano (2004).

${ }^{8}$ Hamao et al. (1990) y Becker et al. (1992).

${ }^{9}$ Malliaris y Urrutia (1992), Jimeno (1995) y Peiró et al. (1998).
} 
cointegración $^{11}, \mathrm{y}$, más recientemente, modelos GARCH multivariante ${ }^{12}$, entre otros.

Asimismo, en los últimos años, han surgido una serie de trabajos que han analizado la influencia que en la interrelación de los mercados bursátiles internacionales han tenido determinados acontecimientos, tales como el establecimiento de la Unión Monetaria Europea ${ }^{13}$, la crisis financiera de $1997^{14} \mathrm{y}$, recientemente, la crisis subprime ${ }^{15}$.

El propósito de este trabajo es analizar el efecto que la reciente crisis financiera ha tenido en la interrelación del mercado bursátil español con los mercados de valores norteamericano, alemán, francés y británico. De la literatura existente se puede concluir que el mercado español está fuertemente interrelacionado con los mercados internacionales. Así pues, la existencia de efectos desbordamiento o spillovers $^{16}$, tanto en el rendimiento como en la volatilidad, entre el mercado español y el resto de mercados está suficientemente evidenciada ${ }^{17}$. Por tanto, la aportación de este trabajo es comprobar si el proceso de transmisión entre el mercado bursátil español y cada uno de los mercados considerados se ha visto afectado, y cómo, por la reciente crisis financiera. Como se ha comentado, las estrategias de inversión y la regulación de los mercados financieros dependen del grado de integración entre los mercados internacionales.

Para llevar a cabo el objetivo del trabajo se va a estimar un modelo VARGARCH bivariante ${ }^{18}$ y asimétrico, que permite recoger los efectos spillover, tanto en la media como en la varianza, de los rendimientos y la existencia de posibles asimetrías en el impacto de los shocks en la volatilidad. Asimismo, también se va a tener en cuenta la posibilidad de que existan cambios estructurales en la varianza de las series, para evitar, como indican Lameroux y Lastra-

${ }^{10}$ Eun y Shim (1989), Peña (1992), Pérez y Torra (1995), Wu y Su (1998) y Climent et al. (1999).

${ }^{11}$ Corhay et al. (1993) y Arshanapalli y Doukas (1993).

${ }^{12}$ Karolyi (1995), Koutmos y Booth (1995), Fratzsher (2002), Worthington y Higgs (2004), Caporale et al. (2006) y Büttner y Hayo (2011).

${ }^{13}$ Fratzscher (2002), Kim et al. (2005) y Büttner y Hayo (2011).

${ }^{14}$ Frexedas y Vayá (2005) y Caporale et al. (2006).

${ }^{15}$ Karim et al. (2011).

${ }^{16}$ Se entiende por efecto desbordamiento o spillover los efectos que un mercado tiene en otro mercado.

${ }^{17}$ Climent et al. (1999), Kim et al. (2005), Aragó y Fernández (2007) y Kenourgios et al. (2009).

${ }^{18}$ El hecho de considerar modelos bivariante, frente a la posibilidad de especificar modelos más complejos se debe a que se está interesado en aislar el efecto total que cada mercado tiene con el mercado español. Los modelos más complejos permitirían obtener el efecto parcial, pero no el efecto total, al descontar los efectos que los mercados tienen entre sí. 
pes (1990), que la excesiva persistencia de la varianza se deba a la existencia, y no consideración, de cambios estructurales en la varianza condicional.

Del modelo VAR-GARCH bivariante se va a obtener una medida del grado de integración del mercado español con los mercados considerados, como es el coeficiente de correlación condicional ${ }^{19}$. El análisis del mismo va a permitir concluir si se produjo o no una variación de la interrelación de los mercados con la crisis subprime, que haya obligado a los agentes económicos a adaptar su comportamiento a la nueva situación.

La estructura del trabajo es como sigue: tras esta introducción se analizan, en el apartado segundo, los datos. A continuación, en el apartado tercero se expone la metodología econométrica utilizada y en el apartado cuarto se presenta la evidencia empírica obtenida. Finalmente, en el apartado quinto, se recogen las principales conclusiones del trabajo.

\section{ANÁLISIS DE LOS DATOS}

En este trabajo se utilizan los datos de apertura y cierre diario de los índices bursátiles de los mercados de New York, Alemania, Reino Unido, Francia y España, durante el período comprendido entre el 3 de enero de 2000 y el 29 de junio de 2012, lo que da lugar a una muestra de 3.246 observaciones. No obstante, dado que no todos los días hay sesiones en todas las bolsas consideradas, la muestra se reduce a 3.039 observaciones, cuando se considera las bolsas española, norteamericana y británica, y a 3.153 observaciones, cuando se analiza conjuntamente la bolsa española y las bolsas alemana y francesa. Los índices considerados han sido los siguientes: el IBEX35 para España, el DAX para Alemania, el CAC40 para Francia, el FTSE100 para el Reino Unido y el SP500 para Estados Unidos ${ }^{20}$. Siguiendo a Dungey et al. (2010), que fijan el comienzo de la crisis generada por las hipotecas subprime el 26 de julio de 2007, el período muestral se ha dividido en dos subperíodos: el período pre crisis que abarca desde el 3 de enero de 2000 hasta el 25 de julio de 2007, y el período posterior que abarca desde el 26 de julio de 2007 al 29 de junio de 2012.

Las series de rendimientos diarios han sido calculadas como la diferencia entre el logaritmo del precio de cierre y de apertura de cada sesión:

$$
R_{i, t}=\log P_{i, t}^{\text {cierre }}-\log P_{i, t}^{\text {apertura }}
$$

donde $\log P_{i, t}$ es el logaritmo del índice bursátil del mercado i en el día t.

\footnotetext{
${ }^{19}$ Chiang et al. (2007) consideran que el coeficiente de correlación condicional obtenido a través de un modelo GARCH multivariante tiene en cuenta directamente la heterocedasticidad de los rendimientos, tal y como indican Forbes y Rigobon (2002).

${ }^{20}$ Los datos han sido obtenidos de Yahoo Finance: http://www.finance.yahoo.com
} 
En la literatura se ha procedido habitualmente de esta forma con el objetivo de solventar el problema de solapamiento entre horarios de negociación de los distintos mercados bursátiles ${ }^{21}$. Hay que tener en cuenta que en las bolsas europeas el solapamiento es total, por lo que cabría esperar que no existiese ese problema. No obstante, esta forma de proceder permite obtener rendimientos diarios para todos los días en los que las bolsas están operativas. Si se obtiene el rendimiento como la diferencia entre los precios de cierre entre dos sesiones consecutivas, los rendimientos no son diarios para las sesiones de los viernes y en las sesiones posteriores a días festivos. Además, al no tener en cuenta las sesiones en las que alguna bolsa ha estado abierta y otras no, si se considera la diferencia al cierre se introduciría esquemas de medias móviles en los datos.

Otra cuestión que se ha tenido en cuenta en la literatura es si se analizan las series de los índices bursátiles en la moneda nacional o se convierten todos los índices a la misma moneda. Li y Majerowska (2008) consideran los índices en moneda local para centrarse solo en las variaciones de los índices y evitar, de esta forma, las distorsiones causadas por la evolución del tipo de cambio de las monedas. Por otro lado, en general, los resultados no parecen verse afectados por esta cuestión ${ }^{22}$, por lo que en este trabajo se van a considerar los índices bursátiles en su moneda nacional.

Siguiendo a Rashid y Ahmad (2008) y Cao y Tsay (1992), la volatilidad del rendimiento diario se estima utilizando la siguiente expresión ${ }^{23}$ :

$$
\sigma_{t}=\sqrt{(\pi / 2)}\left|R_{t}-\mu\right|
$$

donde $\mathrm{R}_{\mathrm{t}}$ es el rendimiento diario para el día t y $\mu$ es la media de la serie.

La Tabla 1 recoge los estadísticos descriptivos de los índices bursátiles ${ }^{24} \mathrm{y}$ de sus rendimientos. Como puede observarse, el estadístico Bera-Jarque indica que ninguna de las series sigue una distribución normal. De los contrastes de raíz unitaria se deduce que no se puede rechazar que los índices bursátiles sean $\mathrm{I}(1)$, frente a la alternativa de ser I(0), mientras que si se rechaza la hipótesis de $\mathrm{I}(1)$, frente a la alternativa de ser I(0), en el caso de los rendimientos.

La Figura 1 recoge la evolución temporal de los índices bursátiles y de sus rendimientos. Del análisis del mismo se observa que tanto la media como la varianza de los índices bursátiles son cambiantes en el tiempo, presentando el comportamiento típico de series con varianza estocástica y, por tanto, no esta-

\footnotetext{
${ }^{21}$ En nuestro caso existe solapamiento de horario entre Estados Unidos y el mercado español.

${ }^{22}$ Ver Hamao et al. (1990), Lee et al. (2004) y Kenourgios et al. (2009).

${ }^{23}$ Se trata de un estimador insesgado de la desviación típica de los rendimientos. Si $\mu=0$, la volatilidad se puede aproximar por el valor absoluto de los rendimientos o por el cuadrado de éstos, tal y como propuso Merton (1980).

${ }^{24} \mathrm{Al}$ cierre del mercado y en logaritmos.
} 
cionarias. Por su parte, los rendimientos presentan un comportamiento de serie estacionaria, observándose que se mueven en torno a un nivel constante e igual a cero $^{25}$, aunque presentan una varianza que no es constante a lo largo del tiempo. Se observa que existen períodos de elevada volatilidad, seguidos de otros en los que la volatilidad es menor. Este tipo de comportamiento se denomina agrupamiento de la volatilidad o clustering de volatilidad, y Bollerslev (1986) propuso la utilización de modelos GARCH para series de datos con estas características.

\section{Tabla 1}

Estadísticos descriptivos de los índices y rendimientos bursátiles

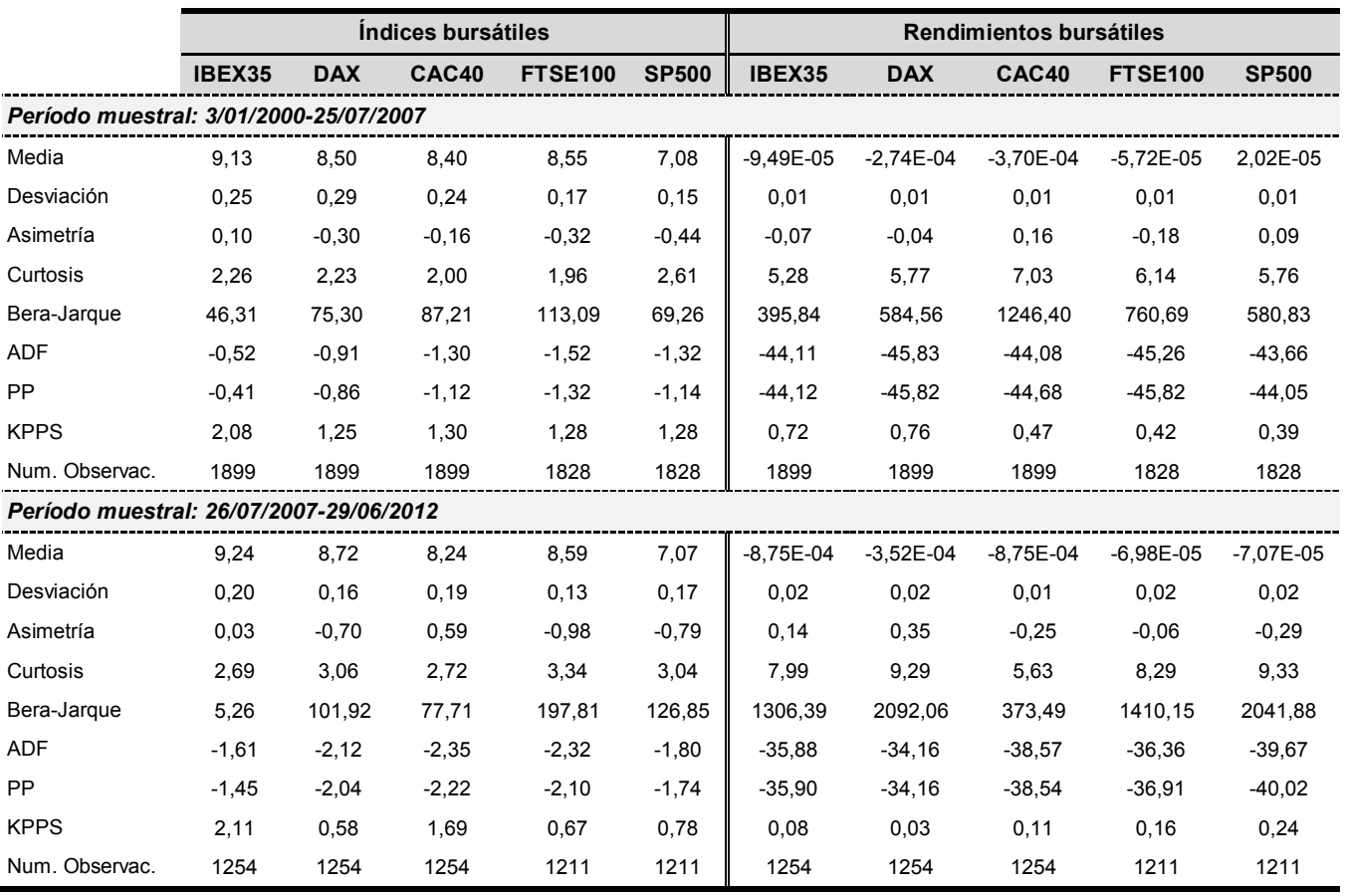

Nota: Los valores críticos de los contraste de ADF y PP son $-3,43,-2,86$ y $-2,57$ al $1 \%$, 5\% y $10 \%$, respectivamente.

Los valores críticos del contraste de KPSS son 0,74, 0,45 y 0,35 al 1\%, 5\% y $10 \%$ respectivamente.

Los valores críticos del contraste de Bera-Jarque son 9,21, 5,99 y 4,61 al 1\%, 5\% y 10\%, respectivamente.

Fuente: Elaboración propia.

${ }^{25} \mathrm{Al}$ calcular el estadístico Media/Desviación, con los datos de la Tabla 1, para los rendimientos se obtiene un $p$-value, para todos los casos, superior a 0.97 , indicando que no se puede rechazar la hipótesis nula de que la media sea distinta de cero. 
Figura 1

Índices y rendimientos bursátiles
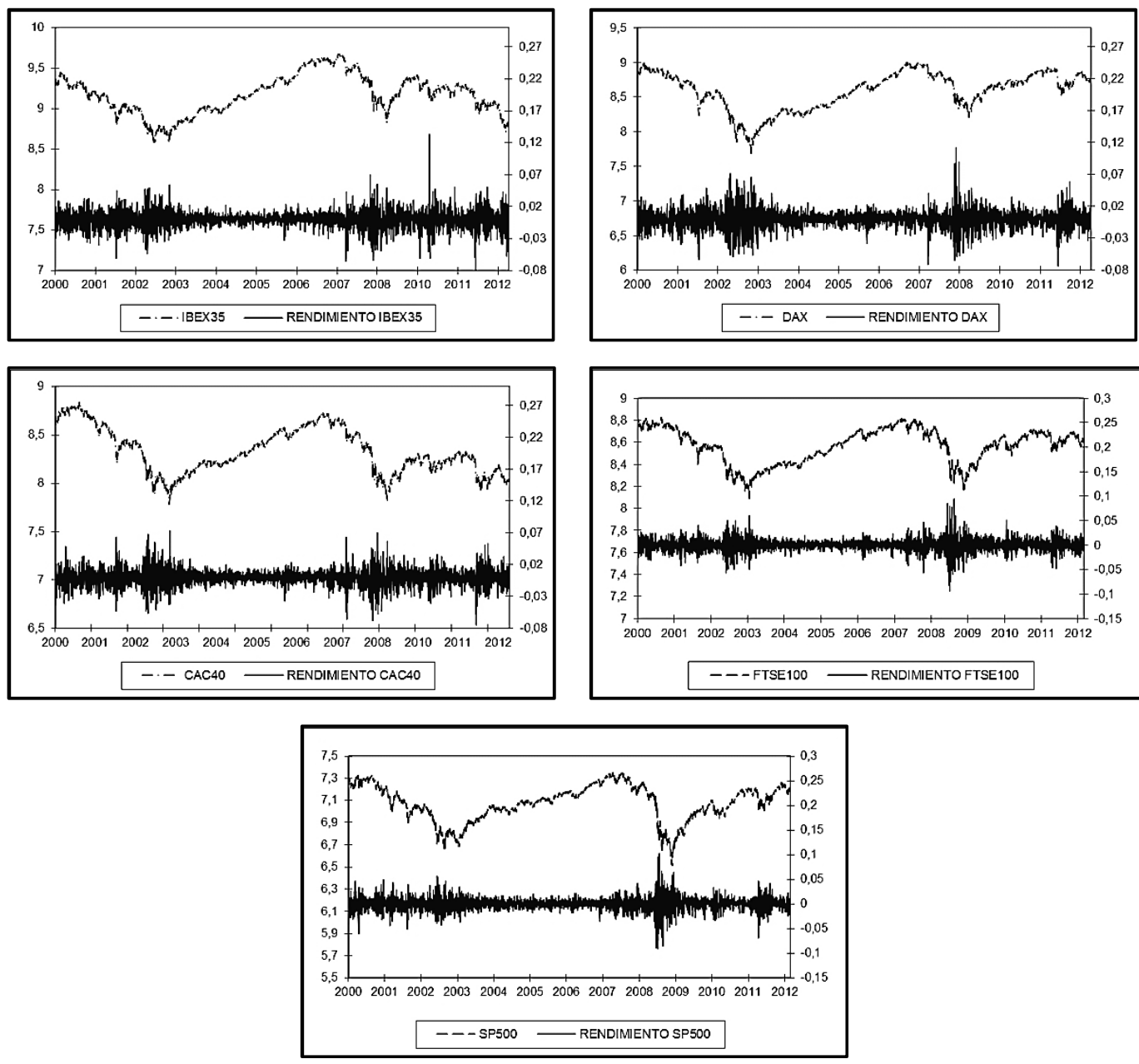

Nota: Los índices bursátiles están medidos en logaritmos.

Fuente: Elaboración propia.

La Tabla 2 presenta las correlaciones cruzadas de los rendimientos y de la volatilidad del mercado español con los rendimientos y volatilidades de los otros mercados considerados. La correlación puede considerarse como una primera aproximación de la relación existente entre los mercados ${ }^{26}$. Como puede comprobarse, se ha considerado también la correlación desfasada un período

\footnotetext{
${ }^{26}$ Isakov y Pérignon (2000).
} 
con el objetivo de comprobar si existe relación con retardo ${ }^{27}$. Los resultados indican que la correlación contemporánea entre los rendimientos es elevada y positiva, indicando que los mercados se mueven conjuntamente en la misma dirección. No obstante, se observa que la correlación contemporánea es más elevada entre el mercado español y los dos mercados de la Unión Monetaria (UM), siendo menor con los otros dos mercados, sobre todo con el mercado

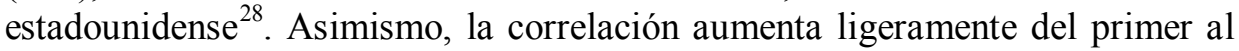
segundo subperíodo con los mercados de valores alemán, francés y estadounidense, mientras que disminuye con el mercado bursátil del Reino Unido. Con respecto a la correlación no contemporánea, ésta es igual a cero o muy reducida.

Tabla 2

Correlaciones muestrales cruzadas

\begin{tabular}{|c|c|c|c|c|c|}
\hline \multirow{2}{*}{\multicolumn{2}{|c|}{ Período muestral: }} & \multicolumn{2}{|c|}{ Rendimientos } & \multicolumn{2}{|c|}{ Volatilidad } \\
\hline & & $\begin{array}{l}03 / 01 / 2000- \\
25 / 07 / 2007 \\
\end{array}$ & $\begin{array}{l}26 / 07 / 2007- \\
29 / 06 / 2012 \\
\end{array}$ & $\begin{array}{l}03 / 01 / 2000- \\
25 / 07 / 2007 \\
\end{array}$ & $\begin{array}{l}26 / 07 / 2007- \\
29 / 06 / 2012 \\
\end{array}$ \\
\hline \multirow{3}{*}{ DAX } & $t-1$ & $-0,0158$ & $-0,0193$ & $0,2646^{*}$ & $0,1051^{*}$ \\
\hline & $t$ & $0,7313^{*}$ & $0,7652^{*}$ & $0,6032^{*}$ & $0,6710^{*}$ \\
\hline & $t+1$ & $-0,0345$ & $-0,0391$ & $0,2434^{\star}$ & $0,0948^{*}$ \\
\hline \multirow{3}{*}{ CAC40 } & $t-1$ & $-0,0279$ & $-0,0587^{*}$ & $0,2661^{*}$ & $0,1119^{*}$ \\
\hline & $t$ & $0,7941^{*}$ & $0,8470^{*}$ & $0,7072^{\star}$ & $0,7392^{*}$ \\
\hline & $t+1$ & $-0,0242$ & $-0,0602^{*}$ & $0,2371^{*}$ & $0,1070^{*}$ \\
\hline \multirow{3}{*}{ FTSE100 } & $t-1$ & $-0,0131$ & $-0,0183$ & $0,2687^{*}$ & $0,1007^{*}$ \\
\hline & $t$ & $0,6734^{*}$ & $0,6363^{*}$ & $0,5551^{*}$ & $0,4951^{*}$ \\
\hline & $t+1$ & $-0,0282$ & $-0,0395$ & $0,2595^{\star}$ & $0,1119^{*}$ \\
\hline \multirow{3}{*}{ SP500 } & $t-1$ & $-0,0493^{*}$ & 0,0251 & $0,1927^{*}$ & $0,1212^{*}$ \\
\hline & $t$ & $0,4735^{*}$ & $0,5323^{*}$ & $0,4347^{\star}$ & $0,4278^{*}$ \\
\hline & $t+1$ & $-0,0143$ & $-0,0504^{\text {** }}$ & $0,2508^{\star}$ & $0,1351^{\star}$ \\
\hline
\end{tabular}

Nota: * Significativo al 5\%. ** Significativo al $10 \%$.

Fuente: Elaboración propia.

Con respecto a la volatilidad, la correlación contemporánea también es elevada y positiva, observándose correlaciones con retardos significativas entre el mercado español y el resto de mercados en ambas direcciones. Al igual que con los rendimientos, se observa que la correlación contemporánea es mayor entre el mercado español y los mercados de la UM, y menor con las bolsas de Estados Unidos y Reino Unido.

La Tabla 3 recoge el test de cointegración de Johansen entre el índice IBEX35 y cada uno de los índices de los mercados bursátiles considerados. Los

${ }^{27}$ Hay que observar que el coeficiente de correlación entre el rendimiento del Ibex35 en t y el rendimiento del mercado foráneo con un lead es equivalente al coeficiente de correlación entre el rendimiento del Ibex35 retardado un período y el rendimiento del mercado foráneo en $\mathrm{t}$.

${ }^{28}$ Este resultado es acorde con el observado por Climent et al. (1999), quienes señalan que la correlación contemporánea aumenta con el solapamiento de horario. 
resultados que aparecen recogidos en el cuadro son los valores de los estadísticos traza y valor propio máximo, para la hipótesis nula de ninguna relación de cointegración, considerándose un retardo ${ }^{29}$. Los resultados obtenidos indican que el IBEX35 no está cointegrado con ningún mercado bursátil, por lo que no existe relación a largo plazo. Este resultado implica que los inversores pueden disminuir a largo plazo el riesgo de su inversión a través de la diversificación, puesto que los mercados no vienen determinados conjuntamente en el largo plazo. No obstante, a pesar de que los mercados no están cointegrados, pueden existir relaciones de causalidad a corto plazo.

Tabla 3

Contraste de cointegración de Johansen

\begin{tabular}{|c|c|c|c|c|c|c|}
\hline & \multicolumn{3}{|c|}{ Período muestral: 3/01/2000-25/07/2007 } & \multicolumn{3}{|c|}{ Período muestral: 26/06/2007-29/06/2012 } \\
\hline & $\begin{array}{l}\text { Valor } \\
\text { Propio }\end{array}$ & $\begin{array}{c}\text { Estadístico } \\
\text { Traza }\end{array}$ & $\begin{array}{l}\text { Estadístico } \\
\text { del Máximo }\end{array}$ & $\begin{array}{c}\text { Valor } \\
\text { Propio }\end{array}$ & $\begin{array}{c}\text { Estadístico } \\
\text { Traza }\end{array}$ & $\begin{array}{l}\text { Estadístico } \\
\text { del Máximo }\end{array}$ \\
\hline DAX & 0,0050 & 9,7239 & 9,5248 & 0,0019 & 2,5367 & 2,3775 \\
\hline CAC40 & 0,0036 & 7,5008 & 6,8297 & 0,0040 & 5,9990 & 5,0511 \\
\hline FTSE100 & 0,0021 & 4,5989 & 3,8944 & 0,0018 & 2,2133 & 2,2128 \\
\hline SP500 & 0,0035 & 6,8487 & 6,4890 & 0,0024 & 2,9080 & 2,8868 \\
\hline
\end{tabular}

Nota: Los valores críticos para el estadístico traza es 12,321 y para el estadístico del máximo es 11,225.

Fuente: Elaboración propia.

En el siguiente apartado se va a especificar un modelo VAR-GARCH bivariante y asimétrico que trata de recoger la dinámica a corto plazo, permitiendo la existencia de efectos spillover o desbordamiento, tanto en el rendimiento como en la volatilidad. La especificación VAR permite recoger la causalidad entre los mercados, esto es la interrelación en media, mientras que la modelización GARCH bivariante permite diferenciar lo que Engle et al. (1990) denominaron heat waves y meteor showers en la volatilidad. Las olas de calor o heat waves recogen las fuentes de volatilidad específicas de cada país, mientras que la lluvia de meteoritos o meteor showers hacen referencia a la transmisión de shocks entre mercados o países diferentes. En la estimación GARCH bivariante, la importancia de los coeficientes cruzados permite concluir si existe evidencia o no de efectos spillover en la volatilidad. Por otro lado, con el objetivo de determinar si se han producido cambios estructurales en la varianza de las series se va a aplicar el algoritmo modificado de Inclan y Tiao (1994), tal y como hace Ewing y Malik (2013). Posteriormente, se incorporan al modelo GARCH variables dummies que recogen los cambios identificados. Finalmente, del modelo estimado se va a obtener el coeficiente de correlación condicional entre el mercado español y cada uno de los mercados bursátiles considerados. El análisis del

${ }^{29}$ Es el número de retardos determinados por el criterio de Schwarz. La especificación no incluye tendencia determinista en los datos. 
mismo va a permitir concluir si ha aumentado la interrelación de los mercados con la crisis subprime.

\section{METODOLOGÍA ECONOMÉTRICA}

El desarrollo de los modelos GARCH multivariantes ha resultado fundamental para analizar las relaciones entre la volatilidad de distintas series temporales. Sin embargo, en la práctica la aplicación de modelos multivariantes no resulta ser una tarea fácil, por el número de parámetros a estimar y por las restricciones necesarias que hay que imponer a la matriz de varianzas-covarianzas. Por esta razón, a lo largo de la literatura han ido surgiendo diferentes especificaciones de modelos GARCH multivariantes, que han tratado de solucionar estos inconvenientes.

$\mathrm{Al}$ igual que en los modelos GARCH univariantes, la modelización multivariante también permite modelizar asimetrías en el impacto que los shocks tienen sobre la volatilidad. En este trabajo se va a especificar un modelo VARGARCH bivariante y asimétrico que permite recoger los efectos spillover, tanto en la media como en la varianza, de los rendimientos y la existencia de posibles asimetrías en los efectos de los shocks.

La expresión (3) recoge los momentos de primer orden de los rendimientos expresados de acuerdo con un modelo VAR bivariante:

$$
\begin{aligned}
& R_{1, t}=\mu_{1}+\phi_{11} R_{1, t-1}+\phi_{12} R_{2, t-1}+\varepsilon_{1, t} \\
& R_{2, t}=\mu_{2}+\phi_{21} R_{1, t-1}+\phi_{22} R_{2, t-1}+\varepsilon_{2, t} \\
& {\left[\begin{array}{l}
\varepsilon_{1, t} \\
\varepsilon_{2, t}
\end{array}\right] \mid I_{t-1} \approx N\left(0, \quad H_{t}\right)}
\end{aligned}
$$

siendo $\mathrm{I}_{\mathrm{t}-1}$ el conjunto de información en $\mathrm{t}-1$ y $\mathrm{H}_{\mathrm{t}}$ la matriz de varianzas-covarianzas.

Se va a considerar que el subíndice 1 hace referencia al mercado español y el subíndice 2 al mercado foráneo. De esta forma, $\phi_{12}$ y $\phi_{21}$ recogen la interacción entre los rendimientos pasados y presentes entre ambos mercados. En particular, $\phi_{12}$ representa la influencia de los rendimientos pasados del mercado foráneo sobre el español y $\phi_{21}$ la influencia de los rendimientos pasados del mercado español sobre el foráneo.

La representación BEKK, propuesta inicialmente por Baba, Engle, Kraft y Kroner (1990), permite la interrelación entre varianzas y covarianzas condicionadas de las series, garantiza que la matriz de varianzas-covarianzas sea defi- 
nida positiva y el número de parámetros a estimar no sea muy elevado en relación a otras especificaciones ${ }^{30}$.

El modelo BEKK asimétrico supone la siguiente especificación para $\mathrm{H}_{\mathrm{t}}$ :

$$
H_{t}=C^{\prime} C+\sum_{i=1}^{q} A_{i} \varepsilon_{t-i} \varepsilon_{t-1}^{\prime} A_{i}^{\prime}+\sum_{i=1}^{p} B_{i} H_{t-1} B_{i}^{\prime}+S \varepsilon_{t-1}^{-} \varepsilon_{t-1}^{-^{\prime}} S^{\prime}
$$

donde $\mathrm{A}_{\mathrm{i}}, \mathrm{B}_{\mathrm{i}}, \mathrm{C}$ y $\mathrm{S}$ son matrices de parámetros $2 \times 2$, C además es definida positiva y triangular, y $\varepsilon_{t-1}^{-}=\min \left(0, \varepsilon_{t-1}\right)$.

Para el caso de $\mathrm{p}=\mathrm{q}=1$, la representación completa para la matriz de varianzas-covarianzas viene dada por la siguiente expresión:

$$
\begin{aligned}
& {\left[\begin{array}{ll}
h_{11, t} & h_{12, t} \\
h_{12, t} & h_{22, t}
\end{array}\right]=\left[\begin{array}{cc}
c_{11} & 0 \\
c_{12} & c_{22}
\end{array}\right]\left[\begin{array}{cc}
c_{11} & c_{12} \\
0 & c_{22}
\end{array}\right]+\left[\begin{array}{ll}
a_{11} & a_{12} \\
a_{21} & a_{22}
\end{array}\right]\left[\begin{array}{cc}
\varepsilon_{11, t-1}^{2} & \varepsilon_{11, t-1} \varepsilon_{22, t-1} \\
\varepsilon_{11, t-1} \varepsilon_{2, t-1} & \varepsilon_{22, t-1}^{2}
\end{array}\right]\left[\begin{array}{ll}
a_{11} & a_{21} \\
a_{12} & a_{22}
\end{array}\right]+} \\
& {\left[\begin{array}{ll}
b_{11} & b_{12} \\
b_{21} & b_{22}
\end{array}\right]\left[\begin{array}{ll}
h_{11, t-1} & h_{12, t-1} \\
h_{12, t-1} & h_{22, t-1}
\end{array}\right]\left[\begin{array}{ll}
b_{11} & b_{21} \\
b_{12} & b_{22}
\end{array}\right]+\left[\begin{array}{ll}
s_{11} & s_{12} \\
s_{21} & s_{22}
\end{array}\right]\left[\begin{array}{cc}
\varepsilon_{11, t-1}^{2-} & \varepsilon_{11, t-1-1}^{-} \varepsilon_{22, t-1}^{-} \\
\varepsilon_{11, t-1}^{-} \varepsilon_{22, t-1}^{-} & \varepsilon_{22, t-1}^{2}
\end{array}\right] } \\
& {\left[\begin{array}{ll}
s_{11} & s_{21} \\
s_{12} & s_{22}
\end{array}\right] }
\end{aligned}
$$

La matriz A recoge los efectos ARCH mientras que la matriz $\mathrm{B}$ recoge los efectos GARCH. Los elementos de la diagonal de ambas matrices recogen los efectos que los shocks propios y la volatilidad propia tienen, respectivamente, sobre la varianza condicional. Estos coeficientes recogerían las olas de calor o heat waves. Sin embargo, los elementos fuera de la diagonal recogen lo que se denominan efectos spillover sobre la varianza, esto es la influencia de un mercado sobre el otro. De esta forma, los coeficientes fuera de la diagonal recogen lo que Engle et al. (1990) denominaron lluvia de meteoritos o meteor showers. Así, $b_{i j}$ representa el efecto que la volatilidad del mercado $\mathrm{j}$ tiene sobre el mercado i, mientras que $a_{i j}$ son los efectos spillover sobre la varianza del mercado $\mathrm{i}$ de shocks producidos en el mercado $\mathrm{j}$. La matriz $\mathrm{S}$ recoge el efecto leverage, esto es el efecto asimétrico que las innovaciones tienen sobre la volatilidad. De esta forma, se tiene en cuenta la posibilidad de que el efecto de un shock negativo (malas noticias) sobre la volatilidad sea mayor que el de un shock positivo de igual magnitud (buenas noticias).

La función de verosimilitud a estimar para el conjunto de parámetros sería la siguiente:

$$
L(\theta)=-T \ln 2 \pi-\frac{1}{2}\left(\sum_{t=1}^{T} \ln \left|H_{t}\right|+\sum_{t=1}^{T} \varepsilon_{t}^{\prime} H_{t}^{-1} \varepsilon_{t}\right)
$$

${ }^{30}$ Por estas razones, la representación BEKK es una de las más utilizadas en la literatura empírica. 
La correlación condicional vendrá dada por la siguiente expresión:

$$
\rho_{12, t}=\frac{h_{12, t}}{\sqrt{h_{11, t} h_{22, t}}}
$$

Para comprobar si se han producido cambios estructurales en la varianza de las series se ha procedido a aplicar, como hacen Ewing y Malik (2013), el algoritmo modificado ICSS (Sansó et al. (2004)). Inclan y Tiao (1994) proporcionan el algoritmo $\operatorname{ICSS}^{31}$ para determinar cambios estructurales en la varianza incondicional, pero este método no es adecuado en procesos dependientes, como los modelos GARCH. En este sentido, Sansó et al. (2004) realizan un ajuste no paramétrico al algoritmo propuesto por Inclan y Tiao.

Siguiendo a Ewing y Malik (2013), el modelo BEKK asimétrico con cambios estructurales vendría especificado de la siguiente forma:

$$
H_{t}=C^{\prime} C+\sum_{i=1}^{q} A_{i} \varepsilon_{t-i} \varepsilon_{i-1}^{\prime} A_{i}^{\prime}+\sum_{i=1}^{p} B_{i} H_{t-1} B_{i}^{\prime}+S \varepsilon_{t-1}^{-} \varepsilon_{t-1}^{-\prime} S^{\prime}+\sum_{i=1}^{n} D_{i} X_{i} X_{i}^{\prime} D_{i}^{\prime}
$$

donde $\mathrm{D}$ es una matriz diagonal $2 \times 2$ de parámetros y $\mathrm{X}$ es un vector columna $2 \times 1$ de variables dummies que recogen los $n$ cambios estructurales de la varianza.

\section{RESULTADOS EMPÍRICOS}

El algoritmo modificado ICSS identifica dos cambios estructurales en la varianza del IBEX35 y del FTSE100. En el caso del IBEX35 los cambios están fechados el 17 de marzo de 2003 y el 20 de febrero de 2007, mientras que en el índice FTSE100 los cambios se producen el 22 de marzo de 2004 y el 5 de mayo de 2007. En el resto de índices no se detectan cambios en la varianza ${ }^{32}$.

La Tabla 4 recoge los resultados de la estimación por máxima verosimilitud del modelo (3) con cambios estructurales en la varianza, para los dos subperíodos considerados ${ }^{33,34}$. Al estimar el modelo se ha tenido especial cuidado en que recogiese correctamente la estructura GARCH de las perturbaciones, por lo que el modelo finalmente seleccionado, en todos los casos, ha sido un VARGARCH $(2,1)$ asimétrico. Los residuos de los modelos estimados se comportan adecuadamente de acuerdo con una batería de contrastes a los que se les ha sometido, y que aparecen recogidos en la Tabla 4.

\footnotetext{
${ }^{31}$ El método se basa en la suma acumulativa de cuadrados de residuos.

${ }^{32}$ Para aplicar el algoritmo modificado de ICSS se ha utilizado el programa RATS.

33 Para la estimación por máxima verosimilitud del modelo (3) se ha utilizado el programa econométrico Eviews.

${ }^{34}$ Los modelos presentados en la Tabla 4 recogen todos los coeficientes estimados, sean o no significativos, tal y como se hace de forma habitual en la literatura, con el objetivo de facilitar la comparación entre los dos subperíodos.
} 
Tabla 4

Estimación modelo VAR-GARCH bivariante y asimétrico con cambios estructurales en la varianza

\begin{tabular}{|c|c|c|c|c|c|c|c|c|}
\hline & \multicolumn{4}{|c|}{ 03/01/2000-25/07/2007 } & \multicolumn{4}{|c|}{ 26/07/2007-29/06/2012 } \\
\hline & DAX & CAC40 & FTSE100 & SP500 & DAX & CAC40 & FTSE100 & SP500 \\
\hline \multicolumn{9}{|c|}{ Panel A: Ecuación Media } \\
\hline$\mu_{1}$ & $0,0007^{*}$ & $0,0008^{*}$ & $0,0007^{*}$ & $0,0007^{\star}$ & 0,0002 & $0,0006^{\star *}$ & 0,0005 & 0,0001 \\
\hline$\mu_{2}$ & $0,0007^{*}$ & $0,0004^{*}$ & $0,0007^{*}$ & $0,0007^{\star}$ & 0,0003 & 0,0004 & $0,0011^{*}$ & $0,0013^{*}$ \\
\hline$\phi_{11}$ & 0,0386 & $0,1209^{*}$ & 0,0089 & $-0,0481^{\text {** }}$ & 0,0102 & $0,0917^{*}$ & $-0,0282$ & $-0,0214$ \\
\hline$\phi_{12}$ & $-0,0798^{*}$ & $-0,1695^{\star}$ & $-0,0454$ & 0,0015 & $-0,0312$ & $-0,1721^{*}$ & $-0,0104$ & $-0,0151$ \\
\hline$\phi_{21}$ & $-0,0265$ & 0,0210 & $-0,0073$ & 0,0306 & $-0,0650^{*}$ & 0,0103 & $-0,0324$ & 0,0225 \\
\hline$\phi_{22}$ & $-0,0298$ & $-0,0896^{\star}$ & $-0,0832^{*}$ & $-0,0719^{*}$ & $0,0735^{\star *}$ & $-0,1016^{*}$ & $-0,0326$ & $-0,1377^{*}$ \\
\hline \multicolumn{9}{|c|}{ Panel B: Ecuación Varianza } \\
\hline $\mathrm{C}_{11}$ & $-0,0014^{*}$ & $-0,0013^{\star}$ & $-0,0010^{\star}$ & $0,0010^{\star}$ & $0,0029^{*}$ & $-0,0027^{\star}$ & $0,0031^{\star}$ & $-0,0039^{*}$ \\
\hline $\mathrm{C}_{12}$ & $-0,0013^{*}$ & $-0,0005^{\star}$ & $-0,0011^{*}$ & $0,0007^{*}$ & $0,0016^{*}$ & $-0,0021^{*}$ & $0,0015^{*}$ & $-0,0007^{*}$ \\
\hline $\mathrm{c}_{22}$ & 1,87E-07 & $0,0008^{*}$ & $0,0006^{*}$ & $0,0012^{*}$ & $0,0007^{\star}$ & $0,0015^{*}$ & $0,0013^{*}$ & $-0,0014^{*}$ \\
\hline$a_{111}$ & $0,3007^{*}$ & $0,2507^{*}$ & 0,0825 & $-0,0423$ & $-0,3043^{*}$ & $0,3003^{*}$ & $0,2046^{*}$ & $-0,2938^{*}$ \\
\hline$a_{112}$ & $-0,1524^{*}$ & $-0,0626$ & $-0,2405^{\star}$ & $-0,1321^{*}$ & $0,1391^{* \star}$ & $-0,3612^{*}$ & $-0,0780$ & $0,2813^{*}$ \\
\hline$a_{121}$ & 0,0745 & $0,1232^{*}$ & $0,1650^{*}$ & $0,1980^{*}$ & $-0,0316$ & 0,0642 & 0,0300 & $-0,0389$ \\
\hline$a_{122}$ & $-0,1113$ & $-0,1028$ & $-0,1810^{*}$ & $-0,1656^{*}$ & $-0,1064$ & $-0,2167^{\star}$ & $-0,1047$ & 0,0185 \\
\hline$a_{211}$ & $-0,0296$ & $-0,1894^{*}$ & $0,2005^{\star}$ & $0,1975^{\star}$ & $-0,0147$ & 0,0496 & $0,1513^{*}$ & $0,2137^{*}$ \\
\hline$a_{212}$ & $0,1748^{*}$ & $0,2895^{*}$ & $-0,0462$ & $-0,1337^{*}$ & $0,2004^{*}$ & 0,1177 & 0,0550 & $0,1727^{*}$ \\
\hline$a_{221}$ & $-0,1277^{*}$ & $-0,0161$ & 0,0606 & $0,0890^{\star *}$ & $-0,0758^{\star *}$ & 0,0213 & $-0,0685^{\star *}$ & $-0,0539^{* *}$ \\
\hline$a_{222}$ & $0,2950^{*}$ & $0,2321^{*}$ & 0,0712 & $0,1298^{*}$ & $0,3182^{*}$ & $0,2421^{*}$ & $0,2841^{*}$ & $0,2831^{*}$ \\
\hline$b_{11}$ & $0,9268^{*}$ & $0,8950^{*}$ & $0,9376^{*}$ & $0,9453^{*}$ & $0,9189^{*}$ & $0,9347^{*}$ & $0,9124^{*}$ & $0,8425^{*}$ \\
\hline$b_{12}$ & $-0,0093$ & $0,0376^{\star *}$ & $-0,0029$ & $-0,0084$ & 0,0013 & $-0,0182$ & 0,0055 & 0,0307 \\
\hline$b_{21}$ & $-0,0130$ & 0,0305 & $-0,0207$ & 0,0287 & 0,0068 & 0,0433 & $-0,0005$ & $-0,0065$ \\
\hline$b_{22}$ & $0,9481^{*}$ & $0,9269^{*}$ & $0,9503^{*}$ & $0,9133^{*}$ & $0,9259^{*}$ & $0,8675^{*}$ & $0,9276^{*}$ & $0,9299^{*}$ \\
\hline $\mathrm{s}_{11}$ & $0,3519^{*}$ & $0,3485^{*}$ & $0,3033^{*}$ & $0,2902^{*}$ & $0,1909^{*}$ & $0,2535^{*}$ & $0,2693^{*}$ & $0,2982^{*}$ \\
\hline $\mathrm{s}_{12}$ & 0,0159 & $-0,0176$ & 0,0597 & $0,0959^{*}$ & $0,2060^{*}$ & $0,2109^{*}$ & $0,1831^{*}$ & $-0,0422$ \\
\hline $\mathrm{s}_{21}$ & $0,1934^{*}$ & $-0,0426$ & $0,1013^{*}$ & 0,0093 & $-0,0962^{*}$ & $-0,017$ & 0,0268 & $0,0611^{* *}$ \\
\hline $\mathrm{s}_{22}$ & $0,1992^{*}$ & $0,3116^{*}$ & $0,3221^{*}$ & $0,3302^{*}$ & $0,3366^{*}$ & $0,3528^{*}$ & $0,3375^{\star}$ & $0,3623^{*}$ \\
\hline \multicolumn{9}{|c|}{ Panel C: Estadísticos } \\
\hline $\log \mathrm{L}$ & 12766,43 & 13242,12 & 12445,72 & 12079,33 & 7834,62 & 7956,42 & 7209,97 & 7187,12 \\
\hline Q1(12) & 5,29 & 6,33 & 5,19 & 6,51 & 6,12 & 7,18 & 3,78 & 4,76 \\
\hline Q2(12) & 12,41 & 11,58 & 11,64 & 19,31 & 5,24 & 6,57 & 4,34 & 7,43 \\
\hline $\mathrm{Q}^{2}(12)$ & 13,48 & 13,50 & 17,10 & 17,14 & 20,72 & 18,34 & 17,43 & 19,00 \\
\hline $\mathrm{Q}^{2}(12)$ & 4,44 & 15,76 & 10,64 & 11,96 & 7,83 & 5,24 & 12,84 & 16,27 \\
\hline Test de Chow & 4897,15 & 4960,95 & 5028,87 & 5117,19 & & & & \\
\hline LR bivariante & 1590,50 & 1926,94 & 1024,22 & 434,43 & 1292,43 & 1655,88 & 629,90 & 430,56 \\
\hline LR no spillover & 57,01 & 109,68 & 63,65 & 123,09 & 12,84 & 50,35 & 7,06 & 88,66 \\
\hline LR simetría & 210,53 & 418,59 & 283,70 & 237,32 & 205,95 & 210,75 & 150,38 & 159,31 \\
\hline
\end{tabular}

Nota: * Significativo al 5\%. ** Significativo al $10 \%$.

$Q(12)$ y $Q^{2}(12)$ son los estadísticos de Ljung-Box para 12 retardos de los residuos y de los residuos al cuadrado, respectivamente.

$L R$ bivariante es el estadístico razón de verosimilitud de la hipótesis del modelo univariante versus bivariante.

$L R$ no spillover y $L R$ simetría son los estadísticos razón de verosimilitud de las hipótesis de no spillover en la varianza $\left(H_{0}: a_{112}=a_{121}=a_{212}=a_{221}=b_{12}=b_{21}=0\right)$ y simetría $\left(H_{0}\right.$ : $s_{11}=s_{12}=s_{21}=s_{22}=0$ ), respectivamente.

Fuente: Elaboración propia.

Con el objetivo de comprobar estadísticamente que se ha producido un cambio estructural en el proceso de transmisión entre los mercados, se ha conside- 
rado, tal y como hacen Candelon y Lütkepohl (2001), una versión del contraste de Chow propuesta por Doornik y Hendry (1997). Esta versión del contraste de Chow, denominada sample-split (SS), supone que se produce un cambio estructural en el momento $\mathrm{T}_{\mathrm{B}}$. Para llevar a cabo el contraste, se estima el modelo con la muestra total de observaciones, $\mathrm{T}$, así como con las $\mathrm{T}_{1}$ primeras observaciones y con las $\mathrm{T}_{2}$ últimas observaciones, donde $\mathrm{T}_{1}<\mathrm{T}_{\mathrm{B}}$ y $\mathrm{T}_{2} \leq \mathrm{T}-\mathrm{T}_{\mathrm{B}}$. Los residuos obtenidos se denominan $\hat{u}_{t}, \hat{u}_{t}^{(1)} \mathrm{y} \hat{u}_{t}^{(2)}$, respectivamente.

Se define $\hat{\Sigma}=T^{-1} \sum_{t=1}^{T} \hat{u}_{t} \hat{u}_{t}^{\prime}, \hat{\Sigma}_{1,2}=T_{1}^{-1} \sum_{t=1}^{T_{1}} \hat{u}_{t} \hat{u}_{t}^{\prime}+T_{2}^{-1} \sum_{t=T-T_{2}}^{T} \hat{u}_{t} \hat{u}^{\prime}, \quad \hat{\Sigma}_{(1)}=T_{1}^{-1} \sum_{t=1}^{T_{1}} \hat{u}_{t}^{(1)} \hat{u}_{t}^{(1)}$ y $\hat{\Sigma}_{(2)}=T_{2}^{-1} \sum_{t=T-T_{2}}^{T} \hat{u}_{t}^{(2)} \hat{u}_{t}^{(2)^{\prime}}$. El estadístico SS del contraste de Chow se obtiene de la siguiente forma:

$$
\lambda_{S S}=\left(T_{1}+T_{2}\right) \log \operatorname{det} \hat{\Sigma}_{1,2}-T_{1} \log \operatorname{det} \hat{\Sigma}_{(1)}-T_{2} \log \operatorname{det} \hat{\Sigma}_{(2)} \approx \chi_{k}^{2}
$$

donde $\mathrm{k}$ es el número de restricciones de la hipótesis nula de estabilidad estructural en los parámetros del modelo. La hipótesis nula se rechaza si el valor del estadístico es mayor que el valor de la chi-cuadrado para un nivel de significación previamente determinado.

El contraste de Chow conlleva el rechazo de la hipótesis nula de estabilidad estructural en los parámetros de los modelos bivariantes seleccionados. Por tanto, la crisis subprime ha supuesto cambios en el proceso de transmisión entre los mercados bursátiles. A continuación se van a comentar dichos cambios.

Al analizar la significatividad de los coeficientes $\phi_{i j}$ se puede concluir que no existe causalidad en ninguna dirección entre España y Estados Unidos y entre España y el Reino Unido. Con respecto a los países de la UM, se evidencia la existencia de spillover unidireccionales de los rendimientos de Francia hacia los del IBEX35, en los dos subperiodos, pero no al contrario. Por su parte, la causalidad entre los mercados de España y Alemania cambia de dirección en el segundo subperíodo. Así, mientras que en el primer subperíodo Alemania causa a España, en el segundo subperíodo es España quien causa a Alemania.

Con respecto a la varianza, hay evidencia de la existencia de efecto leverage en todos los mercados, esto es de asimetría en el efecto que las innovaciones tienen en la volatilidad, de tal forma que las innovaciones negativas tienen un mayor impacto en la volatilidad, que las innovaciones positivas de igual magnitud. Con respecto al efecto leverage cruzado, el coeficiente $s_{12}$ recoge el efecto que las innovaciones negativas de los mercados foráneos tienen sobre la volatilidad del mercado español, mientras que el coeficiente $s_{21}$ recoge el efecto que las innovaciones negativas del mercado español tienen sobre la volatilidad de los mercados foráneos. En el primer subperíodo solo las noticias negativas 
procedentes de Estados Unidos afectaban la volatilidad del mercado español, mientras que en el segundo subperíodo la volatilidad del mercado español viene afectada por las noticias negativas procedentes de Alemania, Francia y Reino Unido. Por tanto, desaparece el efecto leverage de Estados Unidos a España y aparece en sentido contrario, aunque es muy limitado. Por otro lado, con la crisis, las noticias negativas en Europa afectan a la volatilidad del mercado bursátil español. Esto permite concluir que la crisis ha conllevado un cambio en el centro de influencia en el mercado español, de tal forma que el mercado estadounidense deja de ser centro de influencia, en lo que a las noticias negativas se refiere, siendo sustituido por los mercados europeos.

Por lo que respecta a los efectos cruzados de las innovaciones, los coeficientes $\mathrm{a}_{112}$ y $\mathrm{a}_{212}$ recogen los efectos que los shocks de los mercados foráneos tienen en la volatilidad del mercado español, mientras que $\mathrm{a}_{121} \mathrm{y} \mathrm{a}_{221}$ indican los efectos que las innovaciones del mercado español tienen sobre la volatilidad de los mercados foráneos. De los resultados de la estimación de estos coeficientes puede concluirse que existen efectos cruzados en ambos períodos entre España con Estados Unidos y con Alemania. Sin embargo, se producen cambios en los efectos cruzados de España con Reino Unido y con Francia. Así, mientras que en el primer subperíodo se producen efectos cruzados de las innovaciones entre España y Reino Unido, éstos desaparecen en el segundo subperíodo. Por su parte, la evidencia de la existencia de efectos cruzados de las innovaciones entre España y Francia, en ambas direcciones, en el primer subperíodo, desaparece de forma que tan sólo las innovaciones de Francia afectan a España, en el segundo subperíodo, pero no al contrario.

Finalmente, hay que observar que no hay efecto cruzado de las volatilidades entre los mercados en ninguno de los dos subperíodos. Por tanto, no hay efecto contagio en las volatilidades entre los mercados. La volatilidad de los rendimientos sólo viene determinada por la propia volatilidad del período anterior, que, por otro lado, presenta una elevada inercia.

A modo de resumen y conclusión, se puede decir que en la bolsa española, por lo que a su volatilidad se refiere, no existe efectos desbordamientos procedentes de la volatilidad de los mercados europeos considerados, mientras que si existen efectos desbordamiento causados por shocks de los mercados de valores alemán, francés y estadounidense, puesto que con la crisis el efecto desbordamiento procedente del Reino Unido ha desaparecido.

La Tabla 5 recoge los estadísticos descriptivos de los coeficientes de correlación condicional obtenidos con la expresión (7) del apartado anterior. Por su parte, la Tabla 6 presenta los valores medios de las varianzas y de las covarianzas condicionales estimadas, así como de las varianzas y covarianzas muéstrales. Por otro lado, la Figura 2 recoge la evolución temporal de las covarianzas condicionales estimadas en ambos subperíodos. 
Tabla 5

Estadísticos descriptivos de los coeficientes de correlación condicional

\begin{tabular}{|c|c|c|c|c|c|c|c|c|c|c|c|c|}
\hline & \multicolumn{3}{|c|}{ DAX } & \multicolumn{3}{|c|}{ CAC40 } & \multicolumn{3}{|c|}{ FTSE100 } & \multicolumn{3}{|c|}{ SP500 } \\
\hline & $03 / 01 / 2000$ & $03 / 01 / 2000$ & $26 / 06 / 2007$ & $03 / 01 / 2000$ & $03 / 01 / 2000$ & $26 / 06 / 2007$ & $03 / 01 / 2000$ & $03 / 01 / 2000$ & $26 / 06 / 2007$ & $03 / 01 / 2000$ & $03 / 01 / 2000$ & $26 / 06 / 2007$ \\
\hline & $29 / 06 / 2012$ & $25 / 07 / 2007$ & $29 / 06 / 2012$ & $29 / 06 / 2012$ & $25 / 07 / 2007$ & $29 / 06 / 2012$ & $29 / 06 / 2012$ & $25 / 07 / 2007$ & $29 / 06 / 2012$ & $29 / 06 / 2012$ & $25 / 07 / 2007$ & $29 / 06 / 2012$ \\
\hline Media & 0,769 & 0,751 & 0,796 & 0,819 & 0,793 & 0,851 & 0,644 & 0,653 & 0,637 & 0,498 & 0,471 & 0,529 \\
\hline Desviación & 0,077 & 0,074 & 0,097 & 0,064 & 0,073 & 0,057 & 0,088 & 0,073 & 0,107 & 0,121 & 0,087 & 0,130 \\
\hline ADF & $-11,064$ & $-8,502$ & $-7,754$ & $-13,805$ & $-13,744$ & $-8,505$ & $-10,322$ & $-9,372$ & $-7,830$ & $-11,852$ & $-10,836$ & $-8,062$ \\
\hline p-value & 0,000 & 0,000 & 0,000 & 0,000 & 0,000 & 0,000 & 0,000 & 0,000 & 0,000 & 0,000 & 0,000 & 0,000 \\
\hline PP & $-10,687$ & $-8,081$ & $-7,619$ & $-15,874$ & $-13,620$ & $-10,786$ & $-11,661$ & $-9,390$ & $-7,714$ & $-11,893$ & $-14,793$ & $-9,566$ \\
\hline p-value & 0,000 & 0,000 & 0,000 & 0,000 & 0,000 & 0,000 & 0,000 & 0,000 & 0,000 & 0,000 & 0,000 & 0,000 \\
\hline$\rho=1$ & 0,925 & 0,917 & 0,912 & 0,858 & 0,818 & 0,829 & 0,911 & 0,908 & 0,918 & 0,911 & 0,811 & 0,858 \\
\hline$\rho=2$ & 0,859 & 0,848 & 0,823 & 0,757 & 0,673 & 0,714 & 0,847 & 0,823 & 0,852 & 0,829 & 0,722 & 0,777 \\
\hline$\rho=3$ & 0,796 & 0,790 & 0,759 & 0,677 & 0,558 & 0,638 & 0,779 & 0,746 & 0,786 & 0,756 & 0,638 & 0,695 \\
\hline$\rho=4$ & 0,738 & 0,738 & 0,691 & 0,608 & 0,467 & 0,585 & 0,717 & 0,677 & 0,728 & 0,695 & 0,560 & 0,563 \\
\hline$\rho=12$ & 0,464 & 0,469 & 0,429 & 0,340 & 0,188 & 0,326 & 0,370 & 0,274 & 0,428 & 0,371 & 0,193 & 0,339 \\
\hline \multicolumn{2}{|c|}{ Contraste igualdad media } & \multicolumn{2}{|l|}{14,727} & \multicolumn{3}{|c|}{23,602} & \multicolumn{3}{|c|}{$-3,561$} & \multicolumn{3}{|c|}{8,265} \\
\hline \multicolumn{2}{|l|}{$\rho$-value } & \multicolumn{2}{|l|}{0,000} & \multicolumn{3}{|c|}{0,000} & \multicolumn{3}{|c|}{0,000} & \multicolumn{3}{|c|}{0,000} \\
\hline \multicolumn{2}{|c|}{$\begin{array}{l}\text { Contraste igualdad } \\
\text { varianza }\end{array}$} & \multicolumn{2}{|l|}{1,746} & \multicolumn{3}{|c|}{1,625} & \multicolumn{3}{|c|}{1,476} & \multicolumn{3}{|c|}{1,794} \\
\hline$\rho$-value & & \multicolumn{2}{|l|}{0,000} & \multicolumn{3}{|c|}{0,000} & \multicolumn{3}{|c|}{0,000} & \multicolumn{3}{|c|}{0,000} \\
\hline
\end{tabular}

Fuente: Elaboración propia.

De la Tabla 5 se observa que, como ya se veía en la Tabla 2 que recogía las correlaciones cruzadas muéstrales, la correlación es más elevada entre España y los países de la UM, que entre España con Estados Unidos y Reino Unido. Asimismo, se observa que, a excepción de Reino Unido, que presenta una ligera disminución, todos los coeficientes de correlación condicional incrementan en el segundo subperíodo. No solo incrementa su nivel medio, sino que, por lo general aumenta su variabilidad. Por tanto, atendiendo al aumento experimentado por el coeficiente de correlación condicional, y siguiendo a Forbes y Rigobon $(2002)^{35}$, la reciente crisis financiera tuvo un efecto contagio entre los mercados. Otra consecuencia de este resultado es que durante el período de crisis la ventaja de la diversificación internacional disminuyó, puesto que los mercados se encontraban más expuestos a riesgos procedentes de otros mercados.

Por lo que se refiere a las covarianzas condicionales, de la Figura 2 se observa que éstas son siempre positivas y se producen fuertes variaciones en los primeros años de la década de los 2000, a finales de 2008, y a mediados de 2010 y 2011, correspondiendo con la crisis de las empresas tecnológicas, la caída de Lehman Brothers y episodios de crisis de deuda soberana, respectivamente.

${ }^{35}$ Forbes y Rigobon definen contagio entre los mercados como el cambio en el mecanismo de transmisión, que tiene lugar en períodos de elevada incertidumbre, y que conlleva un incremento significativo de la correlación condicional entre mercados. 
Figura 2

Covarianzas condicionales

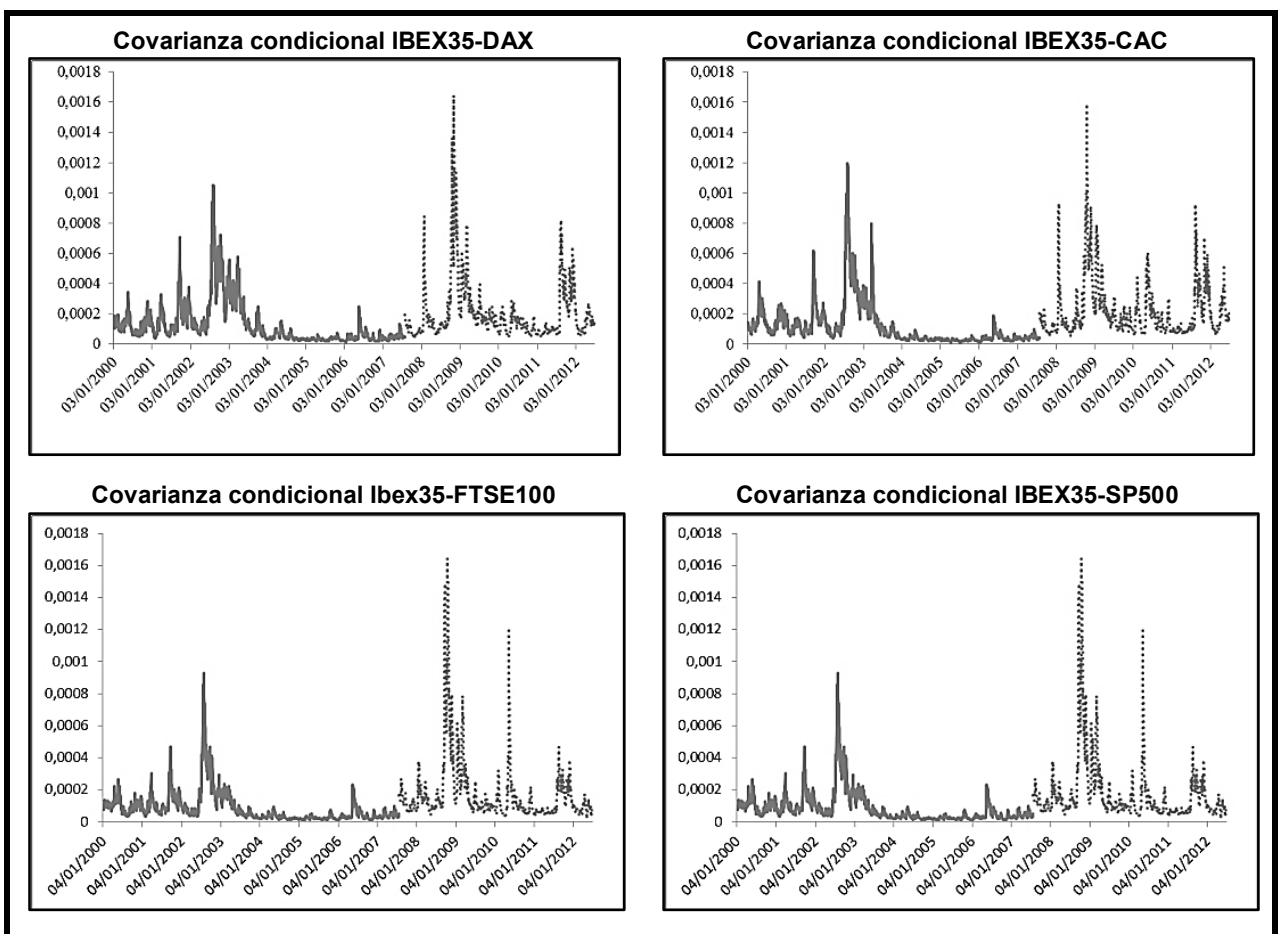

Fuente: Elaboración propia.

De la Tabla 6 se advierte un aumento, en todos los mercados bursátiles, de las varianzas y covarianzas condicionales del primer al segundo subperíodo. Por otro lado, también se comprueba que los valores medios de las varianzas y covarianzas condicionales estimadas por los modelos no se alejan excesivamente de los valores muéstrales.

Con el fin de determinar endógenamente cuando se puede dar por concluida la crisis subprime se aplica el contraste de Bai y Perron (2003) ${ }^{36}$ a un modelo lineal en el que la variable endógena es el coeficiente de correlación condicional del mercado español con cada uno de los mercados considerados y se han considerado dos regresores, una constante y la variable endógena desfasada. Este modelo se ha estimado considerando la matriz de varianzas-covarianzas con-

${ }^{36}$ El contraste de Bai y Perron permite detectar múltiples cambios estructurales en un modelo lineal, que admite formas muy generales de correlación serial, heterocedasticidad, variables endógenas desfasadas, e incluso regresores tendenciales. 
sistente por heterocedasticidad y autocorrelación de Newey y West ${ }^{37}$. En concreto, se quiere comprobar si el contraste de Bai y Perron (BP) detecta cambios en la media del coeficiente de correlación condicional, permitiendo fechar distintas fases tras la crisis subprime. BP sugieren un método secuencial, para determinar el número de cambios estructurales, de tal forma que se minimiza la suma de cuadrados de los residuos. La ventaja del método secuencial de BP, frente a métodos basados en criterios de información, es que considera directamente la presencia de correlación serial y heterocedasticidad en los errores. Los resultados obtenidos del contraste, y presentados en la Tabla 7, permiten situar el inicio del período poscrisis a mediados de 2010.

Tabla 6

Valores medios de las varianzas-covarianzas condicionales y muéstrales

\begin{tabular}{lccc} 
& \multicolumn{3}{c}{ Varianza muestral } \\
\cline { 2 - 4 } & $\mathbf{0 3 / 0 1 / 2 0 0 0}$ & $\mathbf{0 3 / 0 1 / 2 0 0 0}$ & $\mathbf{2 6 / 0 6 / 2 0 0 7}$ \\
DAX & $\mathbf{2 9 / 0 6 / 2 0 1 2}$ & $\mathbf{2 5 / 0 7 / 2 0 0 7}$ & $\mathbf{2 9 / 0 6 / 2 0 1 2}$ \\
\cline { 2 - 4 } CAC40 & 0,000218 & 0,000210 & 0,000230 \\
FTSE100 & 0,000177 & 0,000149 & 0,000218 \\
SP500 & 0,000173 & 0,000125 & 0,000263 \\
IBEX35 & 0,000179 & 0,000124 & 0,000247 \\
& 0,000187 & 0,000129 & 0,000273 \\
\cline { 2 - 4 } & \multicolumn{3}{c}{ Covarianza muestral } \\
\cline { 2 - 4 } & $\mathbf{0 3 / 0 1 / 2 0 0 0}$ & $\mathbf{0 3 / 0 1 / 2 0 0 0}$ & $\mathbf{2 6 / 0 6 / 2 0 0 7}$ \\
DAX & $\mathbf{2 9 / 0 6 / 2 0 1 2}$ & $\mathbf{2 5 / 0 7 / 2 0 0 7}$ & $\mathbf{2 9 / 0 6 / 2 0 1 2}$ \\
\cline { 2 - 4 } CAC40 & 0,000149 & 0,000121 & 0,000192 \\
FTSE100 & 0,000149 & 0,00011 & 0,000206 \\
SP500 & 0,000118 & 0,000086 & 0,000166 \\
\cline { 2 - 4 } & 0,000093 & 0,000060 & 0,000143 \\
\hline
\end{tabular}

\begin{tabular}{ccc}
\hline \multicolumn{3}{c}{ Varianza condicional } \\
\hline $\mathbf{0 3 / 0 1 / 2 0 0 0}$ & $\mathbf{0 3 / 0 1 / 2 0 0 0}$ & $\mathbf{2 6 / 0 6 / 2 0 0 7}$ \\
$\mathbf{2 9 / 0 6 / 2 0 1 2}$ & $\mathbf{2 5 / 0 7 / 2 0 0 7}$ & $\mathbf{2 9 / 0 6 / 2 0 1 2}$ \\
\hline 0,000220 & 0,000211 & 0,000237 \\
0,000184 & 0,000151 & 0,000225 \\
0,000175 & 0,000120 & 0,000248 \\
0,000171 & 0,000121 & 0,000261 \\
0,000202 & 0,000135 & 0,000292 \\
\hline \hline \multicolumn{3}{c}{ Covarianza condicional } \\
\hline $\mathbf{0 3 / 0 1 / 2 0 0 0}$ & $\mathbf{0 3 / 0 1 / 2 0 0 0}$ & $\mathbf{2 6 / 0 6 / 2 0 0 7}$ \\
$\mathbf{2 9 / 0 6 / 2 0 1 2}$ & $\mathbf{2 5 / 0 7 / 2 0 0 7}$ & $\mathbf{2 9 / 0 6 / 2 0 1 2}$ \\
\hline 0,000153 & 0,000124 & 0,000203 \\
0,000157 & 0,000115 & 0,000250 \\
0,000122 & 0,000084 & 0,000173 \\
0,000097 & 0,000059 & 0,000155 \\
\hline
\end{tabular}

Nota: La varianza y covarianza condicional del lbex35 se ha obtenido como la media de los valores estimados para cada uno de los cuatro mercados bursátiles considerados.

Fuente: Elaboración propia.

Finalmente, se estima un modelo ARMA adecuado para cada uno de los coeficientes de correlación condicional del segundo subperíodo, incluyendo dos variables dummies para recoger las dos fases evidenciadas por el contraste de BP. Los resultados, que aparecen recogidos en la Tabla 8 , indican que se produjo un incremento significativo del coeficiente de correlación condicional medio durante la crisis subprime y, a partir de mediados de 2010, aunque los mercados siguen presentando elevada incertidumbre, se produce una reducción de éste. Nuevamente la evidencia es favorable a la hipótesis de contagio durante el período de crisis y está en consonancia con los resultados obtenidos por Nomikos y Salvador (2013), que muestran que la correlación condicional aumenta en períodos de elevado nerviosismo en los mercados.

\footnotetext{
${ }^{37}$ Se ha utilizado el programa econométrico Eviews 8 que permite realizar el contraste de cambio estructural múltiple de Bai y Perron.
} 
Tabla 7

Contraste de cambio estructural de Bai y Perron

\begin{tabular}{|c|c|c|c|}
\hline Correlación & Cambios & Estadístico $\mathrm{F}$ & Punto de Corte \\
\hline \multirow{2}{*}{ España-Alemania } & 0 vs. 1 & 14,35 & \multirow{2}{*}{$18 / 03 / 2010$} \\
\hline & 1 vs. 2 & 3,84 & \\
\hline \multirow{2}{*}{ España-Francia } & 0 vs. 1 & 20,23 & \multirow{2}{*}{ 02/02/2010 } \\
\hline & 1 vs. 2 & 8,28 & \\
\hline \multirow{2}{*}{ España-Reino Unido } & 0 vs. 1 & 23,81 & \multirow{2}{*}{$10 / 02 / 2010$} \\
\hline & 1 vs. 2 & 7,24 & \\
\hline \multirow{2}{*}{ España-Estados Unidos } & 0 vs. 1 & 11,99 & \multirow{2}{*}{$10 / 02 / 2010$} \\
\hline & 1 vs. 2 & 7,98 & \\
\hline
\end{tabular}

Nota: Valores críticos para los contrastes 0 vs. 1 y 1 vs. 2 son 11,47 y 12,95, respectivamente.

Fuente: Elaboración propia.

Tabla 8

Estimación modelos ARMA de los coeficientes de correlación condicional del segundo subperíodo

\begin{tabular}{lcccc}
\hline & DAX & CAC40 & FTSE100 & SP500 \\
\hline \multicolumn{1}{c}{$\boldsymbol{\rho}_{\mathbf{1}}$} & 0,8239 & 0,8635 & 0,6780 & 0,5620 \\
$\boldsymbol{\rho}_{\mathbf{2}}$ & 0,7626 & 0,8370 & 0,5963 & 0,4866 \\
$\boldsymbol{\phi}_{\mathbf{1}}$ & 0,9022 & 0,8918 & 0,8826 & 0,7416 \\
$\boldsymbol{\phi}_{\mathbf{2}}$ & - & - & - & 0,1351 \\
$\boldsymbol{\theta}_{\mathbf{1}}$ & - & $-0,1564$ & - & - \\
\multicolumn{1}{c}{$\boldsymbol{\theta}_{\mathbf{2}}$} & - & $-0,0977$ & - & - \\
\hline Punto de corte & $18 / 03 / 2010$ & $02 / 02 / 2010$ & $10 / 02 / 2010$ & $10 / 02 / 2010$ \\
\hline $\mathbf{R}^{2}$ ajustado & 0,838 & 0,694 & 0,848 & 0,744 \\
$\mathbf{\rho}$ & 0,796 & 0,851 & 0,637 & 0,529 \\
$\mathbf{H}_{\mathbf{0}}: \boldsymbol{\rho}_{\mathbf{1}}=\boldsymbol{\rho}_{\mathbf{2}}$ & & & & \\
$\mathbf{( p - v a l u e )}$ & $(0,0040)$ & $(0,0182)$ & $(0,0000)$ & $(0,0053)$ \\
$\mathbf{D W}$ & 1,980 & 1,993 & 2,063 & 2,044 \\
AIC & $-3,640$ & $-4,061$ & $-3,554$ & $-2,616$ \\
$\mathbf{Q}(\mathbf{1 2})$ & 11,488 & 7,686 & 13,325 & 5,799 \\
$\mathbf{Q}^{2}(\mathbf{1 2})$ & 3,320 & 1,979 & 6,963 & 6,105 \\
\hline
\end{tabular}

Nota: EI FTSE100 y el SP500 incluyen estructura $A R C H$ en la modelización de la varianza de las perturbaciones. DW es el estadístico Durbin y Watson, y $Q$ y $Q^{2}$ son los estadísticos de Ljung-Box para 12 retardos de los residuos y de los residuos al cuadrado, respectivamente.

Fuente: Elaboración propia.

Un análisis más pormenorizado permite concluir que los coeficientes de correlación con el mercado alemán y norteamericano han vuelto a los niveles previos a la crisis subprime, mientras que ha incrementado la interrelación con el mercado francés. Por su parte, con el mercado de valores del Reino Unido se observa una reducción de la interrelación, puesto que el coeficiente de correlación condicional se sitúa en niveles inferiores a los de antes de la crisis. Por tanto, tras la crisis el mercado de valores español está menos afectado por la bolsa de Londres que por la de París, lo que implicará que los inversores al diversificar su inversión contemplaran más al mercado británico que al francés. 


\section{CONCLUSIONES}

Este trabajo analiza la relación a corto y largo plazo entre el mercado bursátil español y los mercados de valores de Estados Unidos, Reino Unido, Alemania y Francia, antes y después de la reciente crisis financiera, con el objetivo de comprobar si se ha producido un cambio en el mecanismo de transmisión entre los mercados. Este trabajo no solo examina la relación de causalidad entre los rendimientos sino también los efectos spillover en la volatilidad.

Los resultados del contraste de Johansen permiten concluir que el mercado español no se encuentra cointegrado a largo plazo con el resto de mercados considerados. Este resultado conlleva la posibilidad de diversificar el riesgo de inversión, puesto que solamente es posible reducir el riesgo cuando los mercados no se encuentran perfectamente correlacionados.

La dinámica a corto plazo se ha recogido a través de un modelo VARGARCH bivariante y asimétrico y los resultados obtenidos permiten concluir que hay efecto leverage en la varianza de los mercados de valores considerados, y que, con la crisis, las noticias negativas en Europa afectan a la volatilidad del mercado bursátil español, mientras que desaparece el efecto leverage de Estados Unidos a España. Por tanto, las malas noticias de los mercados europeos tienen efectos sobre la volatilidad del mercado de valores español pero no las malas noticias procedentes de Estados Unidos.

Al analizar los efectos spillover en la trasmisión de la volatilidad se concluye que no hay efecto contagio entre las volatilidades, de tal forma que un aumento de la volatilidad en un mercado tan sólo le afecta a él mismo, pero no al resto. Este resultado no se ve alterado por la crisis subprime. No obstante, existen efecto contagio de los shocks del mercado norteamericano y alemán al mercado español. Asimismo, con la reciente crisis financiera ha desaparecido el efecto spillover cruzado entre el mercado español y el británico, a la vez que las innovaciones del mercado bursátil español han dejado de influir en la volatilidad del mercado de valores francés, mientras que los shocks de éste último siguen afectando a la volatilidad del mercado español. Por tanto, tras la crisis la volatilidad del mercado de valores español se ve afectado por los shocks que se producen en los mercados alemán, francés y estadounidense.

Por su parte, el análisis del coeficiente de correlación condicional obtenido de la estimación de un modelo VAR-GARCH bivariante y asimétrico permite concluir que en la reciente crisis financiera se produjo un efecto contagio entre los mercados, al aumentar significativamente. El contraste de estabilidad estructural de Bai y Perron ha permitido fechar el final de la crisis subprime a mediados de 2010. Por otro lado, un análisis más pormenorizado del coeficiente de correlación condicional permite concluir que en el período posterior a la crisis subprime, la interrelación del mercado español con el alemán y con el norteamericano ha vuelto a los niveles previos a la crisis, mientras que la inter- 
relación con el mercado francés ha aumentado, reduciéndose, no obstante, la interdependencia con el mercado de valores británico.

\section{REFERENCIAS BIBLIOGRÁFICAS}

AGMON, T. (1972). "The relations among equity markets: A study of share price comovements in the United States, United Kingdom, Germany and Japan". Journal of Finance 27, pp. 839-855.

ARAGÓ, V. y FERNÁNDEZ, M.A (2007)." Influence of structural changes in transmission of information between stock markets: A European empirical study". Journal of Multinational Financial Management 17, pp. 112-124.

ARSHANAPALLI, B. y DOUKAS, J. (1993). "International stock market linkages: Evidence from the Pre- and Post-October 1987 period". Journal of Banking and Finance 17, pp. 193-208.

BABA, Y.; Engel, R.F.; Kraft, D.F. y Kroner, K.F. (1990). "Multivariate simultaneous generalized $A R C H$ ". Mimeo, University of California at San Diego.

BAELE, L.; FERRANDO, A.; HORDAHL, P.; KRYLOVA, E. y MOONET, C. (2004). "Measuring financial integration in the Euro Area". Occasional Paper 14, European Central Bank.

BAI, J. y PERRON, P. (2003). "Computation and analysis of multiple structural change models". Journal of applied econometrics 18, pp. 1-22.

BECKER, K.G, Finnerty, J.E. y Tucker, A.L. (1992). "The intraday interdependence structure between U.S. and Japanese equity markets". Journal of Financial Research 15, pp. 27-37.

BOLLERSLEV, T. (1986). "Generalized autoregressive conditional heteroscedasticity". Journal of Econometrics 31, pp. 309-328.

BÜTTNER, D. y HAYO, B. (2011). "Determinants of European stock market integration". Economic System 35, pp. 574-585.

CAO, C. y TSAY, R.S. (1992). "Nonlinear time-series analysis of stock volatility". Journal of Applied Econometrics, 7, pp. 165-185.

CANDELON, B. y LÜTKEPOHL, H. (2001). "On the reliability of Chow-type tests for parameter constancy in multivariate dynamic models". Economics Letters 73, pp. 155-160.

CAPORALE, G.M.; PITTIS, N. y SPAGNOLO, N. (2006). "Volatility transmission and financial crises". Journal of economics and finance 30, pp. 376-390.

CHIANG, T.C.; JEON, B.N. y Li, H. (2007). "Dynamic correlation analysis of financial contagion: Evidence from Asian markets". Journal of International Money and Finance 26, pp. 1206-1228.

CLIMENT, F.; MENEU, V. y PARDO, A. (1999). "Influencia y sensibilidad de los mercados bursátiles europeos". Información Comercial Española, Revista de Economía 782, pp. 77-87.

CORHAY, A.; RAD, A.T. y URBAIN, J.P. (1993). "Common stochastic trends in European stock markets". Economics Letters 42, pp. 385-390. 
DOORNIK, J.A. y HENDRY, D.F. (1997). Modelling dynamic systems using PcFiml 9.0 for Windows. London: International Thomson Business Press.

DUNGEY, M.; FRY, R.; HERMOSILLO, B.G.; MARTIN, V.L. y TANG, C. (2010). "Are financial crises alike? IMF Working Paper WP10/14, International Monetary Fund, pp. 1-58.

ENGLE, R.F.; ITO, T. y LIN, W.L. (1990). "Meteor showers or heat waves? Heteroskedastic intra-daily volatility in the foreign exchange market". Econometrica 58, pp. 525542.

EUN, C.S. y SHIM, S. (1989). "International transmission of stock market movements". Journal of Financial and Quantitative Analysis 24, pp. 241-256.

EWING, E.T. y MALIK, F. (2013). "Volatility transmission between gold and oil futures under structural breaks". International Review of Economics and Finance 25, pp. 113-121.

FORBES, K.J. y RIGOBON, R. (2002). "No contagion, only interdependence: Measuring stock market co-movements". Journal of Finance 5, pp. 2223-2261.

FREXEDAS, O. y VAYÁ, E. (2005). "Financial Contagion between economies: an exploratory spatial analysis". Estudios de Economía Aplicada, Vol. 23-1, pp. 151-165.

FRATZSCHER, M. (2002). "Financial market integration in Europe: on the effects of EMU on stock markets". International Journal of Finance and Economics 7, pp. 165193.

GRUBEL, H.G. (1968). "Internationally diversified portfolios: welfare gains and capital flows". American Economic Review 58, pp. 1299-1314.

GRUBEL, H.G. y FADNER, K. (1971). "The interdependence of International equity markets". Journal of Finance 26, pp. 89-94.

HAMAO, Y.; MASULIS, R.W. y NG. V. (1990). "Correlations in price changes and volatility across International stock markets". Review of Financial Studies 3, pp. 281307.

INCLAN, C. y TIAO, G.C. (1994). "Use of cumulative sums of squares for retrospective detection of changes of variance". Journal of the American Statistical Association 89, pp. 913-923.

ISAKOV, D. y PÉRIGNON, C. (2000). "On the dynamic interdependence of International stock markets: A Swiss perspective". Zeitschrift für Wolkswirtschaft und Statistik, 136(2), pp. 123-146.

JIMENO, J.P. (1995). "Transmisión de volatilidad: El caso español en la crisis de 1992". Investigaciones Económicas 19, pp. 107-125.

KARIM, B.A.; JAIS, M. y KARIM, S.A.A. (2011). "The subprime crisis and stock index futures markets integration". The Journal of Risk Finance 12, pp. 400-408.

KAROLYI, G.A. (1995). "A multivariate GARCH model of international transmissions of stock returns and volatility: the case of the United States and Canada". Journal of Business and Economics Statistics 13, pp. 5-33.

KENOURGIOS, D.; SAMITAS, A. y PALTALIDIS, N. (2009). "Financial market dynamics in a enlarged European Union". Journal of Economic Integration, 24(2), pp. 197-221.

KIM, S.J.; MOSHIRIAN, F. y WU, E. (2005). "Dynamic stock market integration driven by the European Monetary Union: An empirical analysis". Journal of Banking and Finance 29, pp. 2475-2502. 
KOUTMOS, G. y BOOTH, G. (1995). "Asymmetric volatility transmission in International stock markets". Journal of International Money and Finance 14, pp. 747-762.

LAMOUREUX, C.G., y LASTRAPES, W.D. (1990). "Persistence in variance, structural change and the GARCH model". Journal of Business and Economic Statistics, 8, pp. 225-234.

LEE, B.S.; RUI, O.M. y WANG, S.S. (2004). "Information transmission between the NASDAQ and Asian second board markets". Journal of Banking and Finance 28, pp. 1637-1670.

LESSARD, D.R. (1973). "International portfolio diversification: A multivariate analysis for a group of Latin American countries". Journal of Finance 28, pp.353-364.

LI, H. y MAJEROWSKA, E. (2008). "Testing stock market linkages for Poland and Hungary: A multivariate GARCH approach". Research in International Business and Finance 22, pp. 247-266.

MALLIARIS, A.G. y URRUTIA, J.L. (1992). "The international crash of October 1987: causality tests". Journal of Financial and Quantitative Analysis 27, pp. 353-364.

MERTON, R.C. (1980). "On estimating the expected return on the market, An exploratory investigation". Journal of Financial Economics, 8, pp. 323-364.

NOMIKOS, N. y SALVADOR, W. (2013). "The role of volatility regimes on volatility transmission patterns". forthcoming in Quantitative Finance. Una versión preliminar del trabajo puede encontrarse en http://ssrn.com/abstract=1854403.

PAGANO, M. (1993). "Financial markets and growth: An overview". European Economic Review 37, pp. 613-622.

PRASAD, E.; ROGOFF, K; WEI, S.J. y KOSE, M.A. (2003). "Effects of financial globalization on developing countries: some empirical evidence". International Monetary Fund, Working paper.

PEIRÓ, A.; QUESADA, J. y URIEL, E. (1998). "Transmission of movements in stock markets". The European Journal of Finance 4, pp. 331-343.

PEÑA, J.I. (1992). "Sobre la relación de los mercados bursátiles internacionales y la Bolsa de Madrid”. Información Comercial Española, Revista de Economía 704, pp. 16-24.

PEREZ, J.V. y TORRA, S. (1995). "Transmisión internacional de las rentabilidades y volatilidades entre NYSE e IBEX 35". Cuadernos de Economía 23, pp. 83-101.

RASHID, A. y AHMAD, S. (2008). "Predicting stock returns volatility: An evaluation of linear vs. nonlinear methods". International Research Journal of Finance and Economics, 20, pp. 141-150.

SANSÓ, A.; ARAGÓ, V. y CARRION-i-SILVESTRE, J.L. (2004). "Testing for changes in the unconditional variance of financial time series". Revista de Economía Financiera 4, pp. 32-53.

SORIANO, P. (2004). Transmisión de volatilidad entre mercados financieros". Trabajo de Investigación del Programa de Doctorado Interuniversitario en Finanzas Cuantitativas, No. 3. Universidad de Valencia.

WORTHINGTON, A. y HIGGS, H. (2004). "Transmission of equity returns and volatility in Asian developed and emerging markets: a multivariate GARCH analysis". International Journal of Finance and Economics 9, pp. 71-80.

WU, C. y SU, Y.C. (1998). "Dynamic relations among international stock markets". International Review of Economics and Finance 7, pp. 63-84. 
